No. 569

May 2017

Sequential limiting in continuous and discontinuous Galerkin methods for the Euler equations

V. Dobrev, Tz. Kolev, D. Kuzmin

R. Rieben, V. Tomov

ISSN: 2190-1767 


\title{
Sequential limiting in continuous and discontinuous Galerkin methods for the Euler equations
}

\author{
V. Dobrev ${ }^{\mathrm{a}}$, Tz. Kolev ${ }^{\mathrm{a}}$, D. Kuzmin ${ }^{\mathrm{b}, *}$, R. Rieben ${ }^{\mathrm{c}}$, V. Tomov ${ }^{\mathrm{a}}$ \\ ${ }^{a}$ Center for Applied Scientific Computing, Lawrence Livermore National Laboratory, \\ P.O. Box 808, L-561, Livermore, CA 94551, USA \\ ${ }^{b}$ Institute of Applied Mathematics (LS III), TU Dortmund University, Vogelpothsweg 87, \\ D-44227 Dortmund, Germany \\ ${ }^{c}$ Weapons and Complex Integration, Scientific B-Division, Lawrence Livermore National \\ Laboratory, 7000 East Ave., L-095, Livermore, CA 94551, USA
}

\begin{abstract}
We present a new approach to enforcing local maximum principles in finite element schemes for the compressible Euler equations. In contrast to synchronized limiting techniques for systems of conservation laws, the density, momentum, and total energy are constrained in a sequential manner which guarantees positivity preservation for the pressure and internal energy. After the density limiting step, the total energy and momentum are adjusted to incorporate the irreversible effect of density changes. Then the corresponding antidiffusive corrections are limited to satisfy inequality constraints for the total and kinetic energy. The same element-based limiting strategy is employed in the context of continuous and discontinuous Galerkin methods. The sequential nature of the new limiting procedure makes it possible to achieve crisp resolution of contact discontinuities while using sharp local bounds in the energy constraints. A numerical study is performed for piecewise-linear finite element discretizations of $1 \mathrm{D}$ and 2D test problems.
\end{abstract}

Keywords: systems of conservation laws, finite element methods, local maximum principles, limiting techniques, positivity preservation

\footnotetext{
${ }^{*}$ Corresponding author

Email address: kuzmin@math.uni-dortmund.de (D. Kuzmin)
} 


\section{Introduction}

The accuracy and robustness of finite element methods for the Euler equations of gas dynamics are strongly influenced by the quality of the underlying shock capturing mechanisms. In high-resolution finite element schemes for scalar conservation laws, local maximum principles and nonnegativity constraints can be enforced, e.g., using algebraic limiting techniques based on the flux-corrected transport (FCT) methodology [2, 9, 20, 21] or geometric slope limiters for the derivatives of finite element shape functions $[3,10,11]$. The design of limiting techniques for systems of conservation laws is more involved because preservation of local bounds for the conserved quantities is generally insufficient to prevent spurious oscillations of the velocity, pressure, and other quantities of interest. It is particularly important to enforce positivity preservation for the pressure because negative pressures cause a crash of the simulation run when it comes to calculating the speed of sound.

Positivity-preserving pressure limiters were developed for high-order discontinuous Galerkin discretizations of the Euler equations in a number of recent publications by Zhang and Shu $[30,31]$. Whereas positivity preservation is a necessary condition for obtaining physically realistic solutions, it does not rule out the presence of undershoots and/or overshoots in the pressure distribution. On the other hand, limiting strategies that enforce local maximum principles for derived quantities of interest [14, 23] frequently rely on linearized transformations of variables and cannot guarantee positivity preservation for the pressure and internal energy. This deficiency can be cured by means of a posteriori corrections, as proposed in [6, 14, 29].

Systems of coupled conserved quantities can be limited in a synchronized or sequential manner. Synchronized limiters $[12,14,15,19,21]$ use the same correction factor for all components or a set of balanced correction factors for different components. The latter approach typically leads to constrained optimization problems [16] rather than closed-form expressions. On the other hand, the use of a common correction factor for all conserved quantities tends to produce rather diffusive solutions. In limiting techniques of sequential type, the density correction step is followed by momentum and energy corrections. The bounds for admissible changes in the nodal values of conserved 
quantities may be formulated to constrain changes in derived quantities. In the context of flux-corrected remapping (FCR), limiting techniques of this kind were developed in $[1,27]$. Since the density does not change in the process of subsequent momentum and energy corrections, the sequential approach makes it possible to achieve high resolution of contact discontinuities. However, the density correction may result in violations of local maximum principles for the velocity and specific total energy. A remedy to this problem was recently found by Anderson et al. [1] who have shown that the gradients of low-order approximations to density-weighted variables should be adjusted after the density limiting step to preserve the local bounds.

In this paper, we use bounds-preserving low-order approximations defined as in [1] to develop a new sequential limiting strategy for continuous and discontinuous Galerkin discretizations of the Euler equations. The proposed limiting procedure is designed to constrain the density, specific total energy, and specific kinetic energy. The local bounds for the energy constraints guarantee positivity preservation for the pressure. The kinetic energy limiter is derived using the methodology developed in $[12,19]$ to determine scalar-valued correction factors for momentum changes. A numerical study is performed for $1 \mathrm{D}$ and $2 \mathrm{D}$ test problems discretized using linear finite elements.

\section{Discretization of the Euler equations}

The Euler equations represent a hyperbolic system of conservation laws

$$
\begin{aligned}
\frac{\partial \rho}{\partial t}+\nabla \cdot(\rho \mathbf{v}) & =0 \\
\frac{\partial(\rho \mathbf{v})}{\partial t}+\nabla \cdot(\rho \mathbf{v} \otimes \mathbf{v}+p \mathcal{I}) & =0 \\
\frac{\partial(\rho E)}{\partial t}+\nabla \cdot(\rho E \mathbf{v}+p \mathbf{v}) & =0
\end{aligned}
$$

where $\rho$ is the density, $\mathbf{v}$ is the velocity, $p$ is the pressure, $E$ is the total energy, and $\mathcal{I}$ denotes the identity tensor. The equation of state for an ideal polytropic gas with the heat capacity ratio $\gamma$ reads

$$
p=(\gamma-1)\left(\rho E-\frac{|\rho \mathbf{v}|^{2}}{2 \rho}\right) \text {. }
$$


The system of equations (1)-(3) can be written in the generic form

$$
\frac{\partial \mathcal{U}}{\partial t}+\nabla \cdot \mathcal{F}=0
$$

where

$$
\mathcal{U}=\left[\begin{array}{c}
\rho \\
\rho \mathbf{v} \\
\rho E
\end{array}\right], \quad \mathcal{F}=\left[\begin{array}{c}
\rho \mathbf{v} \\
\rho \mathbf{v} \otimes \mathbf{v}+p \mathcal{I} \\
\rho E \mathbf{v}+p \mathbf{v}
\end{array}\right] .
$$

Inside each element $T^{e}$ of the computational mesh, a finite element approximation $\mathcal{U}_{h}$ to the vector $\mathcal{U}$ of conserved quantities is defined by

$$
\mathcal{U}_{h}^{e}(\mathbf{x})=\sum_{j \in \mathcal{N}^{e}} U_{j}^{e} \varphi_{j}^{e}(\mathbf{x}), \quad \mathbf{x} \in T^{e},
$$

where $\mathcal{N}^{e}$ is the set of nodes for the representation of the polynomial shape function $U_{h}^{e}$ in terms of the local basis functions $\varphi_{j}^{e}$. Here and below, superscripts refer to element numbers and subscripts refer to node numbers.

Variational formulations which lead to algebraic systems for calculating the coefficients $U_{i}^{e}$ in continuous and discontinuous Galerkin (DG) methods can be found in $[13,14,19]$ and $[11,12]$, respectively. The main focus of this work is not on the way in which the Galerkin shape functions $U_{h}^{e}$ are calculated but on postprocessing techniques designed to remove oscillatory antidiffusive components and enforce local maximum principles for selected variables.

In the next sections, we present a unified limiting strategy for continuous and discontinuous Galerkin discretizations based on linear finite elements. Higher order extensions can be constructed using the Bernstein basis representation of the shape functions and localization procedures developed in $[2,20]$ in the context of FEM-FCT algorithms for the linear advection equation.

\section{Limiting for scalar conserved quantities}

Local maximum principles for the density $\rho$ or another scalar quantity $u$ are easy to enforce using a decomposition of the finite element shape function $u_{h}^{e}$ into a monotone low-order component and an antidiffusive correction. Let

$$
u_{i}^{e, H}=u_{h}^{e, H}\left(\mathbf{x}_{i}\right), \quad u_{i}^{e, L}=u_{h}^{e, L}\left(\mathbf{x}_{i}\right)
$$


denote the values of the high-order finite element shape function $u_{h}^{e, H}$ and its low-order counterpart $u_{h}^{e, L}$ at the vertex $\mathbf{x}_{i}$ of element $T^{e}$. A conservative decomposition into antidiffusive element contributions $f_{i}^{e}$ is defined by

$$
m_{i}^{e} u_{i}^{e, H}-m_{i}^{e} u_{i}^{e, L}=f_{i}^{e}, \quad \sum_{i \in \mathcal{N}^{e}} f_{i}^{e}=0,
$$

where $m_{i}^{e}=\int_{T^{e}} \varphi_{i}^{e} \mathrm{~d} \mathbf{x}$ is a diagonal entry of the lumped element mass matrix. The zero sum property of the antidiffusive components implies that

$$
\int_{T^{e}} u_{h}^{e, H} \mathrm{~d} \mathbf{x}=\sum_{i \in \mathcal{N}^{e}} m_{i}^{e} u_{i}^{e, H}=\sum_{i \in \mathcal{N}^{e}} m_{i}^{e} u_{i}^{e, L}=\int_{T^{e}} u_{h}^{e, L} \mathrm{~d} \mathbf{x} .
$$

Hence, the approximations $u_{h}^{e, H}$ and $u_{h}^{e, L}$ have the same mass in element $T^{e}$.

\subsection{Generic element-based limiter}

In the process of limiting, each antidiffusive element contribution is multiplied by a solution-dependent correction factor $\alpha^{e} \in[0,1]$ such that

$$
u_{i}^{\min } \leq u_{i}^{e}=u_{i}^{e, L}+\frac{\alpha^{e} f_{i}^{e}}{m_{i}^{e}} \leq u_{i}^{\max }
$$

where the bounds $u_{i}^{\min }$ and $u_{i}^{\max }$ are defined using the coefficients of the loworder approximation (see below). Note that multiplication by $\alpha^{e}$ preserves the zero sum property of $f_{i}^{e}$ and corresponds to limiting the slope of the finite element shape function without changing the cell average.

To satisfy local maximum principles (10) for all nodes of element $T^{e}$, we define the element-based correction factor $\alpha^{e}$ as follows:

$$
\alpha^{e}=\min _{i \in \mathcal{N}^{e}} \begin{cases}\min \left\{1, \frac{m_{i}^{e}\left(u_{i}^{\max }-u_{i}^{e, L}\right)}{f_{i}^{e}}\right\} & \text { if } f_{i}^{e}>0, \\ 1 & \text { if } f_{i}^{e}=0, \\ \min \left\{1, \frac{m_{i}^{e}\left(u_{i}^{\min }-u_{i}^{e, L}\right)}{f_{i}^{e}}\right\} & \text { if } f_{i}^{e}<0 .\end{cases}
$$

This limiting strategy is common to element-based flux-corrected transport (FCT) algorithms for continuous finite elements [5, 20] and slope limiters for DG methods $[10,11]$. The difference between the continuous and discontinuous versions lies in the definition of the low-order shape functions, antidiffusive element contributions, and bounds for the inequality constraints. 


\subsection{Continuous Galerkin methods}

In the continuous version $[5,20]$ of the above predictor-corrector method, the low-order scheme for calculating $u_{i}^{L}$ is derived by modifying the element matrices of the Galerkin discretization. The element mass matrix $M^{e}$ is replaced by its lumped counterpart $\tilde{M}^{e}=\operatorname{diag}\left\{m_{i}^{e}\right\}$ and the element matrix $K^{e}$ of the discrete transport operator is modified by adding a discrete diffusion operator $D^{e}$. The bounds for inequality constraints (10) are defined by

$$
u_{i}^{\min }=\min _{j \in \mathcal{N}_{i}} u_{j}^{L}, \quad u_{i}^{\max }=\max _{j \in \mathcal{N}_{i}} u_{j}^{L},
$$

where $\mathcal{N}_{i}$ is the set of nodes belonging to the same elements as node $i$.

Since the finite element approximations $u_{h}^{H}$ and $u_{h}^{L}$ are continuous, the nodal values $u_{i}^{e, H}$ and $u_{i}^{e, L}$ are independent of $e \in \mathcal{E}_{i}$, where $\mathcal{E}_{i}$ denotes the set of elements containing node $i$. However, the corrected one-sided limits $u_{i}^{e}$ may attain different values in different elements. Therefore, the final step of the localized element-based FCT algorithm for continuous finite elements $[5,19]$ is the computation of the mass-weighted convex average

$$
u_{i}=\frac{\sum_{e \in \mathcal{E}_{i}} m_{i}^{e} u_{i}^{e}}{\sum_{e \in \mathcal{E}_{i}} m_{i}^{e}}
$$

which lies in the range $\left[u_{i}^{\min }, u_{i}^{\max }\right]$ of admissible values provided that all one-sided limits $u_{i}^{e}$ are constrained to satisfy conditions (10).

\subsection{Discontinuous Galerkin methods}

In the context of a piecewise-linear DG approximation [10, 11], we have

$$
\begin{gathered}
u_{h}^{e, L} \equiv \bar{u}^{e, L}=\frac{1}{\left|T^{e}\right|} \int_{T^{e}} u_{h}^{H} \mathrm{~d} \mathbf{x}=\frac{\sum_{i \in \mathcal{N}^{e}} m_{i}^{e} u_{i}^{e, H}}{\sum_{i \in \mathcal{N}^{e}} m_{i}^{e}} \\
u_{i}^{\min }=\min _{e \in \mathcal{E}_{i}} \bar{u}^{e, L}, \quad u_{i}^{\max }=\max _{e \in \mathcal{E}_{i}} \bar{u}^{e, L}, \\
f_{i}^{e}=m_{i}^{e} \nabla u_{h}^{e, H} \cdot\left(\mathbf{x}_{i}-\overline{\mathbf{x}}^{e}\right), \quad \overline{\mathbf{x}}^{e}=\frac{1}{\left|T^{e}\right|} \int_{T^{e}} \mathbf{x} \mathrm{d} \mathbf{x} .
\end{gathered}
$$

The use of these definitions in (11) leads to the vertex-based version of the Barth-Jespersen limiter [3] for linear shape functions. Hierarchical extensions to higher-order finite elements can be found in $[10,11]$. 


\section{Limiting for systems of conservation laws}

Let us now consider a system of conservation laws for the components of

$$
U_{i}^{e}=\left(\rho_{i}^{e},\left(\rho u_{1}\right)_{i}^{e}, \ldots,\left(\rho u_{m}\right)_{i}^{e}\right)^{T}
$$

and constrain the corresponding antidiffusive element contributions

$$
F_{i}^{e}=\left(f_{i}^{e, \rho}, f_{i}^{e, \rho u_{1}}, \ldots, f_{i}^{e, \rho u_{m}}\right)^{T}
$$

to be local extremum diminishing w.r.t. low-order values of $\rho, u_{1}, \ldots, u_{m}$.

Following Anderson et al. [1], we use the algorithm presented in Section 3 to limit the density $\rho$ before limiting the product variables $\rho u_{1}, \ldots, \rho u_{m}$. Using formula (11) to calculate the correction factor $\alpha^{e, \rho}$, we obtain

$$
\rho_{i}^{e}=\rho_{i}^{e, L}+\frac{\alpha^{e, \rho} f_{i}^{e, \rho}}{m_{i}^{e}} \in\left[\rho_{i}^{\min }, \rho_{i}^{\max }\right]
$$

After the density limiting step, conserved quantities of the form $\rho u$ can be constrained using the strategy proposed in [1] in the context of multi-material remap algorithms. The local maximum principle to be enforced reads

$$
u_{i}^{\min } \leq u_{i}^{e}=\frac{(\rho u)_{i}^{e}}{\rho_{i}^{e}} \leq u_{i}^{\max }
$$

where $\rho_{i}^{e}$ is given by (19). Note that the value of $u_{i}^{e}$ corresponding to $(\rho u)_{i}^{e, L}$ may violate these inequality constraints if the bounds are defined in terms of the low-order nodal values $u_{j}^{e, L}=(\rho u)_{j}^{e, L} / \rho_{j}^{e, L}$. Therefore, we define

$$
u_{i}^{\min }:=\min _{e \in \mathcal{E}_{i}} \bar{u}^{e, L}, \quad u_{i}^{\max }:=\max _{e \in \mathcal{E}_{i}} \bar{u}^{e, L}
$$

as in (15) using the compatible low-order approximation [1]

$$
\bar{u}^{e, L}=\frac{\sum_{i \in \mathcal{N}^{e}} m_{i}^{e}(\rho u)_{i}^{e, L}}{\sum_{i \in \mathcal{N}^{e}} m_{i}^{e} \rho_{i}^{e}}=\frac{\sum_{i \in \mathcal{N}^{e}} m_{i}^{e}(\rho u)_{i}^{e, L}}{\sum_{i \in \mathcal{N}^{e}} m_{i}^{e} \rho_{i}^{e, L}}=\frac{\int_{T^{e}}(\rho u)_{h}^{L} \mathrm{~d} \mathbf{x}}{\int_{T^{e}} \rho_{h}^{L} \mathrm{~d} \mathbf{x}}=\frac{\overline{(\rho u}^{e, L}}{\bar{\rho}^{e, L}}
$$

such that

$$
u_{i}^{\min } \leq \bar{u}^{e, L} \leq u_{i}^{\max }
$$


The unconstrained high-order approximation $\bar{u}_{i}^{e, H}$ can be written as

$$
(\rho u)_{i}^{e, H}=\rho_{i}^{e} \bar{u}^{e, L}+\frac{g_{i}^{e, \rho u}}{m_{i}^{e}},
$$

where

$$
g_{i}^{e, \rho u}=f_{i}^{e, \rho u}+m_{i}^{e}(\rho u)_{i}^{e, L}-m_{i}^{e} \rho_{i}^{e} \bar{u}^{e, L}
$$

is the raw antidiffusive element contribution associated with the low-order approximation $\rho_{i}^{e} \bar{u}^{e, L}$. By definition (22) of $\bar{u}^{e, L}$, we have

$$
\sum_{i \in \mathcal{N}^{e}} g_{i}^{e, \rho u}=\underbrace{\sum_{i \in \mathcal{N}^{e}} f_{i}^{e, \rho u}}_{=0}+\sum_{i \in \mathcal{N}^{e}} m_{i}^{e}(\rho u)_{i}^{e, L}-\bar{u}^{e, L} \sum_{i \in \mathcal{N}^{e}} m_{i}^{e} \rho_{i}^{e}=0 .
$$

Hence, the zero sum property of the antidiffusive components is preserved. Multiplying $g_{i}^{e, \rho u}$ by a correction factor $\alpha^{e, \rho u} \in[0,1]$, we constrain

$$
(\rho u)_{i}^{e}=\rho_{i}^{e} \bar{u}^{e, L}+\frac{\alpha^{e, \rho u} g_{i}^{e, \rho u}}{m_{i}^{e}}
$$

to satisfy (20) or, equivalently, the local maximum principle

$$
\rho_{i}^{e} u_{i}^{\min } \leq(\rho u)_{i}^{e} \leq \rho_{i}^{e} u_{i}^{\max }
$$

These inequality constraints can be enforced using the formula

$$
\alpha^{e, \rho u}=\min _{i \in \mathcal{N}^{e}} \begin{cases}\min \left\{1, \frac{m_{i}^{e} \rho_{i}^{e}\left(u_{i}^{\max }-\bar{u}^{e, L}\right)}{g_{i}^{e, \rho u}}\right\} & \text { if } g_{i}^{e, \rho u}>0, \\ 1 & \text { if } g_{i}^{e, \rho u}=0 \\ \min \left\{1, \frac{m_{i}^{e} \rho_{i}^{e}\left(u_{i}^{\min }-\bar{u}^{e, L}\right)}{g_{i}^{e, \rho u}}\right\} & \text { if } g_{i}^{e, \rho u}<0\end{cases}
$$

The constrained shape function admits the following representation:

$$
\begin{aligned}
(\rho u)_{h}^{e} & =\rho_{h}^{e} \bar{u}^{e, L}+\alpha^{e, \rho u}\left((\rho u)_{h}^{e, H}-\rho_{h}^{e} \bar{u}^{e, L}\right) \\
& =\rho_{h}^{e, L} \bar{u}^{e, L}+\alpha^{\rho}\left(\rho_{h}^{e, H}-\rho_{h}^{e, L}\right) \bar{u}^{e, L}+\alpha^{e, \rho u}\left((\rho u)_{h}^{e, H}-\rho_{h}^{e} \bar{u}^{e, L}\right) .
\end{aligned}
$$

In the case $\rho \equiv$ const, this product limiter reduces to the DG version of (11) in which the low-order values and bounds are defined by cell averages. 


\section{Limiting for the Euler equations}

In applications to the Euler equations of gas dynamics, the generic product limiter presented in Section 4 can be used to enforce local bounds for the specific total energy $E$ and velocity $\mathbf{v}$. Using the same correction factor for all velocity components, we obtain the constrained approximations

$$
\begin{gathered}
(\rho E)_{i}^{e}=\rho_{i}^{e} \bar{E}^{e, L}+\frac{\alpha^{e, \rho E} g_{i}^{e, \rho E}}{m_{i}^{e}} \\
(\rho \mathbf{v})_{i}^{e}=\rho_{i}^{e} \overline{\mathbf{v}}^{e, L}+\frac{\alpha^{e, \rho \mathbf{v}} \mathbf{g}_{i}^{e, \rho \mathbf{v}}}{m_{i}^{e}}
\end{gathered}
$$

The raw antidiffusive element contributions corresponding to

$$
\bar{E}^{e, L}=\frac{\sum_{i \in \mathcal{N}^{e}} m_{i}^{e}(\rho E)_{i}^{e, L}}{\sum_{i \in \mathcal{N}^{e}} m_{i}^{e} \rho_{i}^{e, L}}, \quad \overline{\mathbf{v}}^{e, L}=\frac{\sum_{i \in \mathcal{N}^{e}} m_{i}^{e}(\rho \mathbf{v})_{i}^{e, L}}{\sum_{i \in \mathcal{N}^{e}} m_{i}^{e} \rho_{i}^{e, L}}
$$

are defined by

$$
\begin{aligned}
& g_{i}^{e, \rho E}=f_{i}^{e, \rho E}+m_{i}^{e}(\rho E)_{i}^{e, L}-m_{i}^{e} \rho_{i}^{e} \bar{E}^{e, L} \\
& \mathbf{g}_{i}^{e, \rho \mathbf{v}}=\mathbf{f}_{i}^{e, \rho \mathbf{v}}+m_{i}^{e}(\rho \mathbf{v})_{i}^{e, L}-m_{i}^{e} \rho_{i}^{e} \overline{\mathbf{v}}^{e, L}
\end{aligned}
$$

Since the velocity $\mathbf{v}$ is a vector field, the imposition of local maximum principle on its components would violate the principle of frame indifference [22]. Therefore, it is more appropriate to formulate velocity constraints in terms of the kinetic energy $k=\frac{1}{2}|\mathbf{v}|$. Moreover, the limiting procedure should ensure positivity preservation for the pressure defined by the equation of state

$$
p_{i}^{e}=(\gamma-1)\left((\rho E)_{i}^{e}-\frac{\left|(\rho \mathbf{v})_{i}^{e}\right|^{2}}{2 \rho_{i}^{e}}\right)
$$

where $(\rho E)_{i}^{e}$ and $(\rho \mathbf{v})_{i}^{e}$ are given by (30) and (31), respectively.

The proposed sequential limiter is based on the energy constraints

$$
\begin{aligned}
\rho_{i}^{e} E_{i}^{e, \min } & \leq(\rho E)_{i}^{e} \leq \rho_{i}^{e} E_{i}^{e, \max } \\
\rho_{i}^{e} k_{i}^{e, \min } & \leq \frac{\left|\rho \mathbf{v}_{i}^{e}\right|^{2}}{2 \rho_{i}^{e}} \leq \rho_{i}^{e} k_{i}^{e, \max } .
\end{aligned}
$$


Introducing the compatible low-order approximation

$$
\bar{k}^{e, L}=\frac{\left|\overline{\mathbf{v}}^{e, L}\right|^{2}}{2}
$$

to the kinetic energy, we define the bounds as follows:

$$
\begin{aligned}
& E_{i}^{e, \min }=\max \left\{\bar{k}^{e, L}, \min _{e^{\prime} \in \mathcal{E}_{i}} \bar{E}^{e^{\prime}, L}\right\}, \quad E_{i}^{e, \max }=\max _{e^{\prime} \in \mathcal{E}_{i}} \bar{E}^{e^{\prime}, L}, \\
& k_{i}^{e, \min }:=\min _{e^{\prime} \in \mathcal{E}_{i}} \bar{k}^{e^{\prime}, L}, \quad k_{i}^{e, \max }:=\min \left\{\frac{(\rho E)_{i}^{e}}{\rho_{i}^{e}}, \max _{e^{\prime} \in \mathcal{E}_{i}} \bar{k}^{e^{\prime}, L}\right\},
\end{aligned}
$$

where $(\rho E)_{i}^{e}$ is given by (30). The corresponding correction factor $\alpha^{e, \rho E}$ is calculated using (29) to enforce the total energy constraints (36).

In view of (31) and (38), the constrained kinetic energy can be written as

$$
\frac{\left|\rho \mathbf{v}_{i}^{e}\right|^{2}}{2 \rho_{i}^{e}}=\rho_{i}^{e} \bar{k}^{e, L}+\frac{P_{i}^{e}}{m_{i}^{e}},
$$

where

$$
P_{i}^{e}=\alpha^{e, \rho \mathbf{v}} \overline{\mathbf{v}}^{e, L} \cdot \mathbf{g}_{i}^{e, \rho \mathbf{v}}+\frac{\left(\alpha^{e, \rho \mathbf{v}}\right)^{2}}{2} \frac{\left|\mathbf{g}_{i}^{e, \rho \mathbf{v}}\right|^{2}}{m_{i}^{e} \rho_{i}^{e}}
$$

Substituting (41) into (37), we obtain the equivalent inequality constraints

$$
Q_{i}^{e,-}:=m_{i}^{e} \rho_{i}^{e}\left(k_{i}^{e, \min }-\bar{k}^{e, L}\right) \leq P_{i}^{e} \leq m_{i}^{e} \rho_{i}^{e}\left(k_{i}^{e, \max }-\bar{k}^{e, L}\right)=: Q_{i}^{e,+} .
$$

Note that $k_{i}^{e, \max } \geq \bar{k}^{e, L}$ since $(\rho E)_{i}^{e} \geq \rho_{i}^{e} E_{i}^{e, \min } \geq \rho_{i}^{e} \bar{k}^{e, L}$ by (39). Hence, $Q_{i}^{+, e} \geq 0$ and the kinetic energy constraints are satisfied for $\alpha^{e, \rho \mathbf{v}}=0$.

A quasi-optimal upper bound for admissible correction factors $\alpha^{e, \rho \mathbf{v}}$ can be obtained using the methodology developed in $[12,19]$. The derivation is as follows: For $\alpha^{e, \rho \mathbf{v}} \leq R_{i}^{e} \leq 1$, we use the fact that $\left(\alpha^{e, \rho \mathbf{v}}\right)^{2} \leq \alpha^{e, \rho \mathbf{v}}$ to estimate the quadratic term in the antidiffusive increment (42). This yields

$$
R_{i}^{e} P_{i}^{e,-} \leq P_{i}^{e} \leq R_{i}^{e} P_{i}^{e,+}
$$

where

$$
\begin{aligned}
& P_{i}^{e,+}=\max \left\{0, \overline{\mathbf{v}}^{e, L} \cdot \mathbf{g}_{i}^{e, \rho \mathbf{v}}+\frac{1}{2} \frac{\left|\mathbf{g}_{i}^{e, \rho \mathbf{v}}\right|^{2}}{m_{i}^{e} \rho_{i}^{e}}\right\} \\
& P_{i}^{e,-}=\min \left\{0, \overline{\mathbf{v}}^{e, L} \cdot \mathbf{g}_{i}^{e, \rho \mathbf{v}}\right\} .
\end{aligned}
$$


It follows that conditions (43) can be enforced using the correction factor

$$
\alpha^{e, \rho \mathbf{v}}=\min _{i \in \mathcal{N}^{e}}\left\{\begin{array}{cl}
R_{i}^{e,+} & \text { if } P_{i}^{e,+}>0, P_{i}^{e,-}=0, \\
\min \left\{R_{i}^{e,+}, R_{i}^{e,-}\right\} & \text { if } P_{i}^{e,+}>0, P_{i}^{e,-}<0, \\
R_{i}^{e,-} & \text { if } P_{i}^{e,+}=0, P_{i}^{e,-}<0, \\
1 & \text { if } P_{i}^{e,+}=0, P_{i}^{e,-}=0
\end{array}\right.
$$

where

$$
R_{i}^{e,+}=\min \left\{1, \frac{Q_{i}^{e,+}}{P_{i}^{e,+}}\right\}, \quad R_{i}^{e,-}=\min \left\{1, \frac{Q_{i}^{e,-}}{P_{i}^{e,-}}\right\} .
$$

Indeed, this definition of the nodal correction factors $R_{i}^{ \pm}$implies that

$$
\begin{aligned}
& P_{i}^{e,+}>0, \quad \alpha^{e, \rho \mathbf{v}} \leq R_{i}^{e,+} \quad \Rightarrow \quad P_{i}^{e} \leq R_{i}^{e,+} P_{i}^{e,+} \leq Q_{i}^{e,+} \\
& P_{i}^{e,-}<0, \quad \alpha^{e, \rho \mathbf{v}} \leq R_{i}^{e,-} \quad \Rightarrow \quad P_{i}^{e} \geq R_{i}^{e,-} P_{i}^{e,-} \geq Q_{i}^{e,-} .
\end{aligned}
$$

In summary, the proposed sequential limiting strategy is as follows:

1. Calculate $\rho_{i}^{e}$ using density bounds of the form (12) or (15) and the correction factor $\alpha^{e, \rho}$ defined by (11) in formula (19);

2. Calculate $(\rho E)_{i}^{e}$ using definition (39) of the total energy bounds and the correction factor $\alpha^{e, \rho E}$ defined by (29) in formula (30);

3. Calculate $(\rho \mathbf{v})_{i}^{e}$ using definition (40) of the kinetic energy bounds and the correction factor $\alpha^{e, \rho \mathbf{v}}$ defined by (47) in formula (31);

4. In the CG version: calculate the mass-weighted averages using (13).

Positivity preservation for the pressure $p_{i}^{e}$ defined by (35) is guaranteed since

$$
(\rho E)_{i}^{e} \geq \rho_{i}^{e} k_{i}^{e, \max } \geq \frac{\left|\rho \mathbf{v}_{i}^{e}\right|^{2}}{2 \rho_{i}^{e}}
$$

by (40) and (37). Nonnegativity of the pressure $p\left(U_{i}\right)$ corresponding to the final nodal values $U_{i}=\left(\rho_{i},(\rho \mathbf{v})_{i},(\rho E)_{i}\right)^{T}$ of a continuous finite element approximation $\mathcal{U}_{h}$ can be shown as in [18]. Since the pressure $p(U)$ is a concave function of the conserved variables, Jensen's inequality yields

$$
p\left(U_{i}\right)=p\left(\frac{\sum_{e \in \mathcal{E}_{i}} m_{i}^{e} U_{i}^{e}}{\sum_{e \in \mathcal{E}_{i}} m_{i}^{e}}\right) \geq \frac{\sum_{e \in \mathcal{E}_{i}} m_{i}^{e} p_{i}^{e}}{\sum_{e \in \mathcal{E}_{i}} m_{i}^{e}} \geq 0 .
$$


In addition to positivity preservation, the pressure $p_{i}^{e}$ defined by the nodal equation of state (35) satisfies the local maximum principle

$$
p_{i}^{e, \min } \leq p_{i}^{e} \leq p_{i}^{e, \max }
$$

where

$$
\begin{aligned}
& p_{i}^{e, \max }=(\gamma-1) \rho_{i}^{e}\left(E_{i}^{e, \max }-k_{i}^{e, \min }\right), \\
& p_{i}^{e, \min }=(\gamma-1) \rho_{i}^{e}\left(E_{i}^{e, \min }-k_{i}^{e, \max }\right) .
\end{aligned}
$$

The implied pressure constraints (53) are more realistic than those defined in terms of (compatible) low-order pressures and restrictive enough to prevent spurious ripples that do not violate the global positivity constraint.

\section{Numerical examples}

In this section, we solve standard test problems for the Euler equations using linear $\left(P_{1}\right)$ finite elements and the new sequential limiting procedure. The low-order scheme for the CG version of the element-based FCT algorithm is constructed using Rusanov-type scalar dissipation [19]. In the DG version, we use the Rusanov (local Lax-Friedrichs) or HLL flux approximation on interelement boundaries. Characteristic boundary conditions are imposed weakly using internal traces and freestream values in approximate Riemann solvers for the boundary terms. For a detailed description of the employed CG and DG discretizations, we refer the reader to $[11,12,13,14,19]$.

In all numerical examples of this section, we use an explicit third-order strong stability preserving (SSP) Runge-Kutta time stepping method [7, 8, 12]. The employed mesh size and time step are listed in the caption to each figure. The presented DG results for all test problems were calculated using a 2D code on a uniform mesh of square cells. For visualization purposes, we $L^{2}$-project DG solutions into the space of continuous bilinear finite elements.

For the sake of comparison, we also present numerical solutions obtained using the synchronized limiters developed in $[12,19]$. These limiters constrain the products $\rho E$ and $\rho p$ using bounds that depend on the range of admissible density values and become less restrictive in regions where $\rho$ is smooth. 


\subsection{Shock tube problem}

Sod's shock tube problem [26] is a well-known benchmark for the Euler equations. The computational domain $\Omega=(0,1)$ has reflective walls and is initially separated by a membrane into two sections. When the membrane is removed, the gas begins to flow into the region of lower pressure. The initial condition for the nonlinear Riemann problem is given by

$$
\left[\begin{array}{l}
\rho_{L} \\
\mathbf{v}_{L} \\
p_{L}
\end{array}\right]=\left[\begin{array}{l}
1.0 \\
0.0 \\
1.0
\end{array}\right], \quad\left[\begin{array}{l}
\rho_{R} \\
\mathbf{v}_{R} \\
p_{R}
\end{array}\right]=\left[\begin{array}{c}
0.125 \\
0.0 \\
0.1
\end{array}\right] .
$$

The removal of the membrane at the time $t=0$ releases a shock wave that propagates to the right with velocity satisfying the Rankine-Hugoniot conditions. All of the primitive variables are discontinuous across the shock which is followed by a contact discontinuity. The latter represents a moving interface between the regions of different densities but constant velocity and pressure. The rarefaction wave propagates in the opposite direction providing a smooth transition to the original values of the state variables in the left part of the domain. Hence, the one-dimensional flow pattern in the shock tube is characterized by three waves traveling at different speeds.

The density (blue), velocity (green), and pressure (red) distributions displayed in Figs 1 and 2 correspond to the final time $t=0.231$. The exact solution is shown by the solid lines without markers. The corresponding finite element approximations are shown as solid lines with bullet markers. For a better quantitative comparison of the methods under investigation, we list the numerical errors $E_{1} \approx\left\|u-u_{h}\right\|_{L^{1}(\Omega)}$ above each diagram.

Figure 1 presents the diffusive low-order solution and constrained Galerkin approximations based on a continuous piecewise-linear discretization. It can be seen that antidiffusive corrections lead to significant improvements but the choice of the limiting strategy is important. Segregated limiting of the conserved quantities $(\rho, \rho v, \rho E)$ is ideally suited for simulation of contact discontinuities (see Fig. 1(b)) but may allow violations of local bounds for primitive variables. Limiters designed to constrain the pressure or kinetic energy are less likely to produce undershoots and overshoots in other fields. The results presented in Figs 1(c) and (d) were obtained using the synchronized $(\rho, E, p)$ limiter developed in [19] and the sequential $(\rho, E, k)$ limiter 
(a) $E_{1}=(2.58,4.89,2.29) \cdot 10^{-2}$

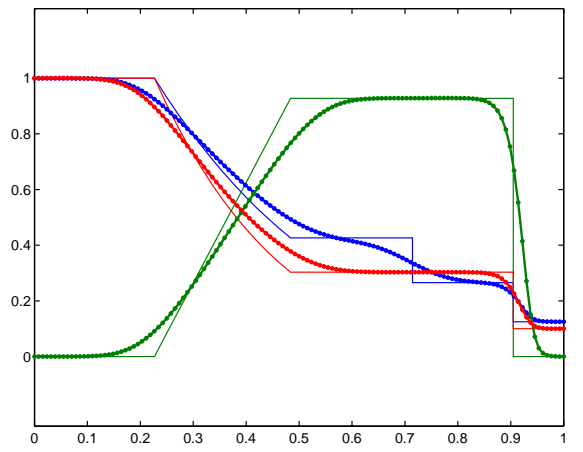

(c) $E_{1}=(0.70,1.46,0.57) \cdot 10^{-2}$

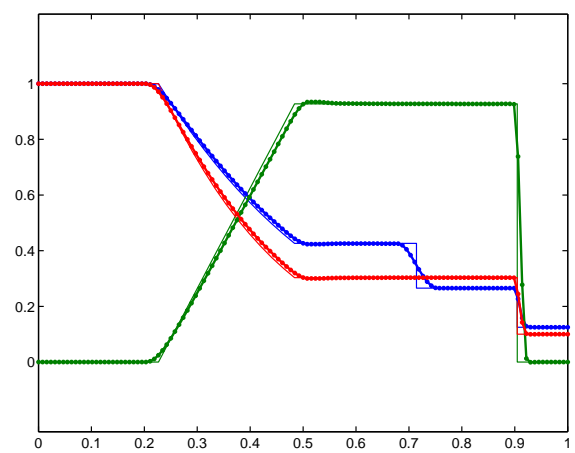

(b) $E_{1}=(0.80,1.68,0.66) \cdot 10^{-2}$

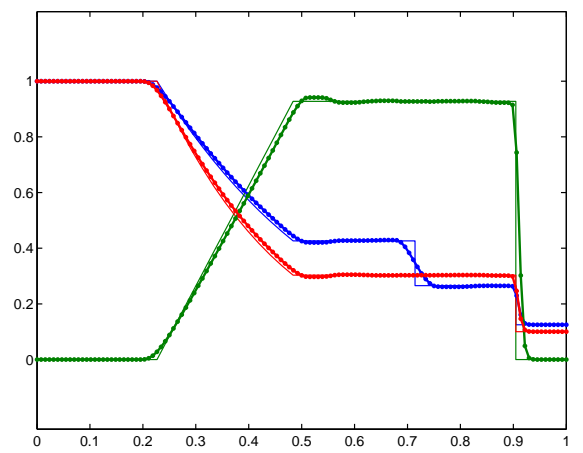

(d) $E_{1}=(0.95,2.07,0.82) \cdot 10^{-2}$

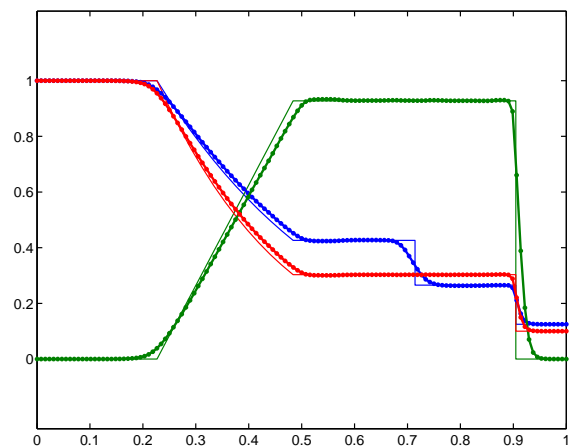

Figure 1: Sod's shock tube: $(\rho, v, p)$ at $t=0.231, h=\frac{1}{128}, \Delta t=10^{-3}$, CG version, (a) low-order Rusanov approximation, (b) segregated limiting of $(\rho, \rho v, \rho E)$, (c) synchronized limiting of $(\rho, E, p)$, (d) sequential limiting of $(\rho, E, k)$.

presented in Section 5. The accuracy of the constrained solutions is comparable in this particular test. The local bounds for the synchronized version are generally less restrictive, which explains the smaller absolute $L^{1}$ errors and better preservation of the contact discontinuity. However, the use of slack bounds in energy and pressure constraints may lead to stronger spurious distortions of solution profiles in some applications (see below).

The numerical solutions presented in Fig. 2 were calculated using the 2D implementation of the DG method. It can be seen that the piecewise-constant $\left(P_{0}\right)$ discretization based on the Rusanov (RUS) flux is far more diffusive than that based on the HLL flux approximation. The difference between the 
(a) $E_{1}=(2.52,4.59,2.22) \cdot 10^{-2}$

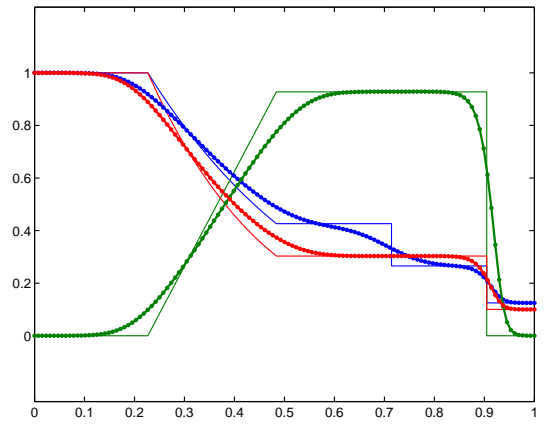

(c) $E_{1}=(4.04,8.99,3.19) \cdot 10^{-3}$

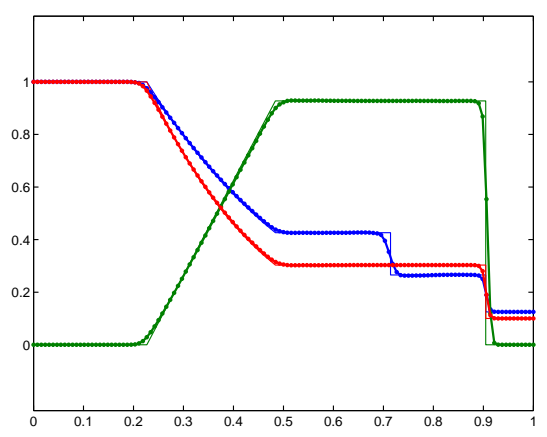

(b) $E_{1}=(1.97,3.67,1.70) \cdot 10^{-2}$

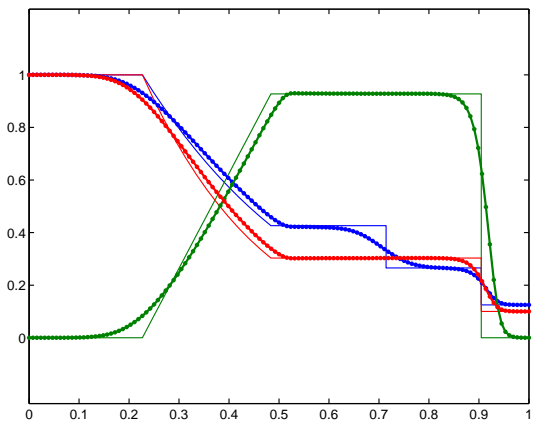

(d) $E_{1}=(3.78,8.54,2.97) \cdot 10^{-3}$

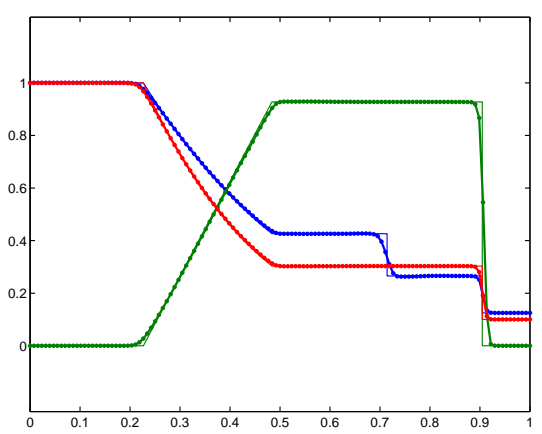

Figure 2: Sod's shock tube: $(\rho, v, p)$ at $t=0.231, h=\frac{1}{128}, \Delta t=10^{-3}$, DG version, (a) RUS- $P_{0}$, (b) HLL- $P_{0}$, (c) limited RUS- $P_{1}$, (d) limited HLL- $P_{1}$.

corresponding piecewise-linear $\left(P_{1}\right)$ solutions is less pronounced. The DG version of the new sequential limiter produces nonoscillatory solutions which are more accurate than the corresponding CG results in Fig. 1(d).

\subsection{Blast wave problem}

The blast wave problem of Woodward and Colella [28] is a far more challenging test. The flow of a gamma-law gas, with $\gamma=1.4$, takes place between reflecting walls, and the initial condition consists of the three constant states

$$
\left[\begin{array}{l}
\rho_{L} \\
v_{L} \\
p_{L}
\end{array}\right]=\left[\begin{array}{c}
1.0 \\
0.0 \\
1000.0
\end{array}\right], \quad\left[\begin{array}{l}
\rho_{M} \\
v_{M} \\
p_{M}
\end{array}\right]=\left[\begin{array}{c}
1.0 \\
0.0 \\
0.01
\end{array}\right], \quad\left[\begin{array}{l}
\rho_{R} \\
v_{R} \\
p_{R}
\end{array}\right]=\left[\begin{array}{c}
1.0 \\
0.0 \\
100.0
\end{array}\right]
$$

associated with $\Omega_{L}=(0,0.1), \Omega_{M}=(0.1,0.9)$, and $\Omega_{R}=(0.9,1)$. 
The above initial conditions give rise to two strong blast waves which eventually collide. The flow evolution involves complex interactions of shocks, rarefactions, and contact discontinuities in a small region of space. These interactions are particularly difficult to capture using FCT algorithms which tend to clip peaks and distort steep fronts within the local bounds. The latter phenomenon is known as terracing. It can be alleviated by prelimiting the fluxes or improving the phase accuracy of the high-order scheme [29].

The density distributions shown in Figs 3 and 4 correspond to the final time $t=0.038$. The solution produced by the synchronized $(\rho, E, p)$ limiter

(a) $E_{1}=1.32 \cdot 10^{-1}$

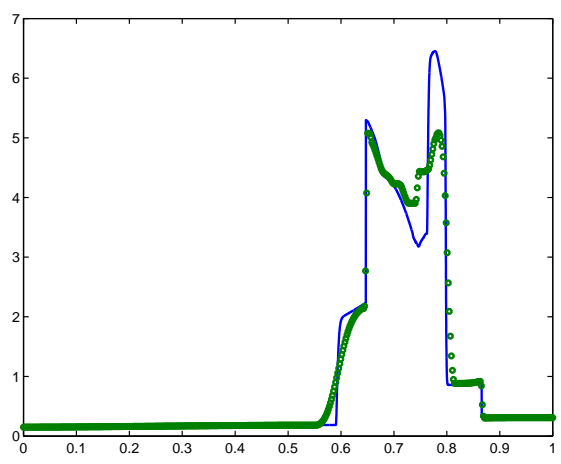

(b) $E_{1}=1.00 \cdot 10^{-1}$

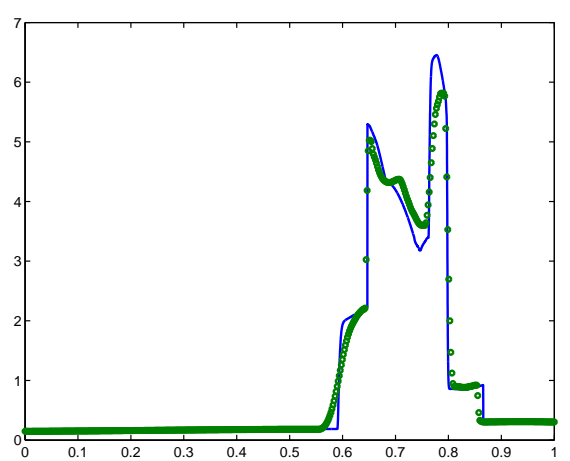

Figure 3: Blast wave problem: $\rho$ at $t=0.038, h=\frac{1}{512}, \Delta t=10^{-5}, \mathrm{CG}$ version, (a) synchronized limiting of $(\rho, E, p)$, (b) sequential limiting of $(\rho, E, k)$.

(a) $E_{1}=8.81 \cdot 10^{-2}$

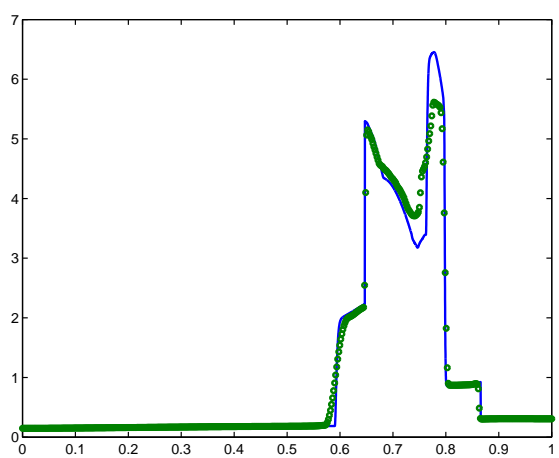

(b) $E_{1}=6.21 \cdot 10^{-2}$

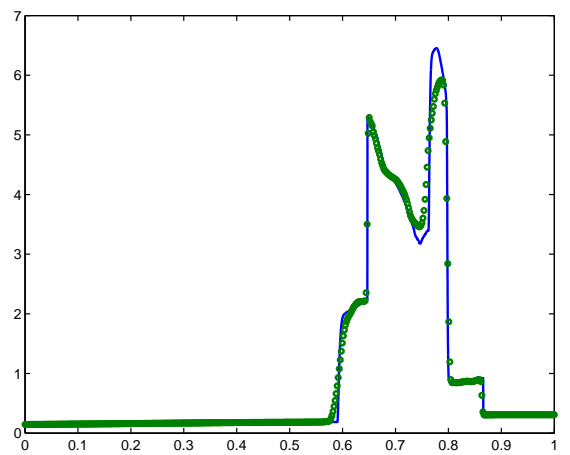

Figure 4: Blast wave problem: $\rho$ at $t=0.038, h=\frac{1}{512}, \Delta t=10^{-5}$, DG-HLL version, (a) synchronized limiting of $(\rho, E, p)$, (b) sequential limiting of $(\rho, E, k)$. 
exhibits strong clipping and terracing effects. The better performance of the new sequential $(\rho, E, k)$ limiter is due to more realistic energy constraints. As before, the DG-HLL version is more accurate than the CG version. However, higher accuracy comes at the cost of using more degrees of freedom.

\subsection{Double Mach reflection}

In the next example, we consider the double Mach reflection benchmark [28] for the two-dimensional Euler equations. The computational domain for this test is the rectangle $\Omega=(0,4) \times(0,1)$. The flow pattern features a propagating Mach 10 shock in air $(\gamma=1.4)$ which initially makes a $60^{\circ}$ angle with a reflecting wall. The following pre-shock and post-shock values of the flow variables are used to define the initial and boundary conditions

$$
\left[\begin{array}{c}
\rho_{L} \\
u_{L} \\
v_{L} \\
p_{L}
\end{array}\right]=\left[\begin{array}{c}
8.0 \\
8.25 \cos \left(30^{\circ}\right) \\
-8.25 \sin \left(30^{\circ}\right) \\
116.5
\end{array}\right], \quad\left[\begin{array}{l}
\rho_{R} \\
u_{R} \\
v_{R} \\
p_{R}
\end{array}\right]=\left[\begin{array}{c}
1.4 \\
0.0 \\
0.0 \\
1.0
\end{array}\right] .
$$

Initially, the post-shock values (subscript $L$ ) are prescribed in the subdomain $\Omega_{L}=\{(x, y) \mid x<1 / 6+y / \sqrt{3}\}$ and the pre-shock values (subscript $R$ ) in $\Omega_{R}=\Omega \backslash \Omega_{L}$. The reflecting wall corresponds to $1 / 6 \leq x \leq 4$ and $y=0$. No boundary conditions are required along the line $x=4$. On the rest of the boundary, the post-shock conditions are assigned for $x<1 / 6+(1+20 t) / \sqrt{3}$ and the pre-shock conditions elsewhere. The so-defined values along the top boundary describe the exact motion of the initial Mach 10 shock.

In Figs 5 and 6, we present snapshots of the density and pressure distribution at $t=0.2$ calculated using the $P_{0}$ and $P_{1}$ discontinuous Galerkin methods. As expected, the results obtained using the Rusanov flux are more diffusive than those produced by the HLL version. The $P_{1}$ approximations constrained using the new sequential $(\rho, E, k)$ limiter exhibit sharp resolution of small-scale features and are free of spurious oscillations. We conclude that limiting techniques of this kind are well suited for shock capturing in finite element methods for computational gas dynamics. Numerical studies of synchronized positivity-preserving limiters for DG and CG approximations to the double Mach reflection problem were performed in $[12,14,19]$. Since no reference solution is available for this benchmark problem, we abstain from a quantitative comparison of the results produced by different limiters. 
(a) RUS- $P_{0}$

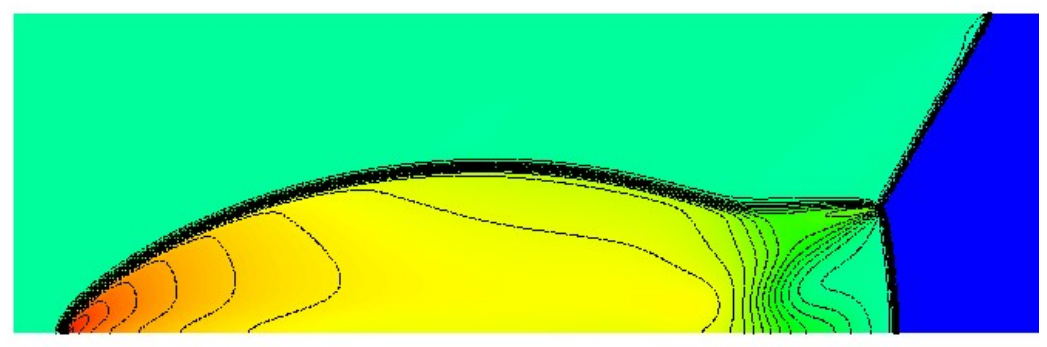

(b) HLL- $P_{0}$

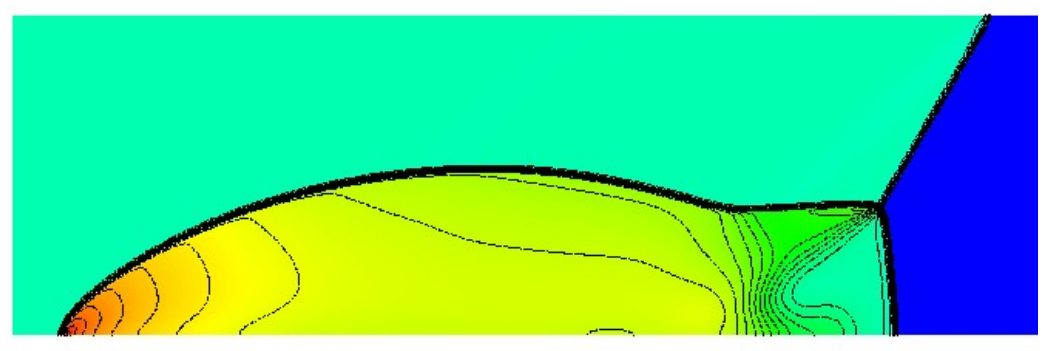

(c) RUS- $P_{1}$

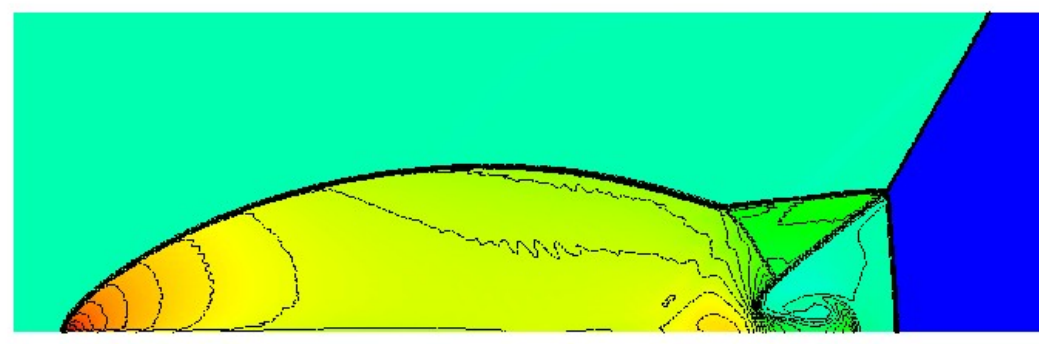

(d) HLL- $P_{1}$

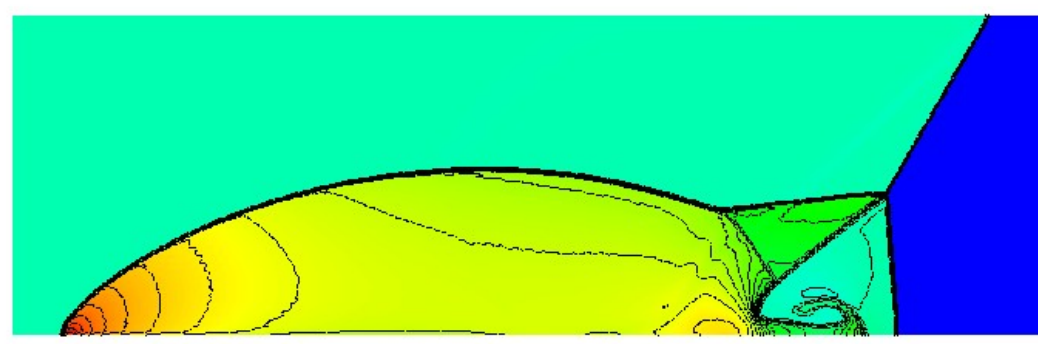

Figure 5: Double Mach reflection: $\rho$ at $t=0.2$, DG version, $h=\frac{1}{256}, \Delta t=10^{-4}$. 
(a) RUS- $P_{0}$

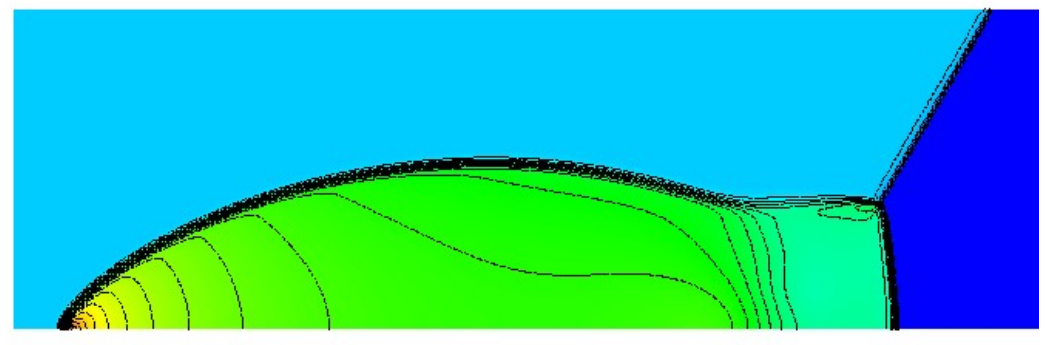

(b) HLL- $P_{0}$

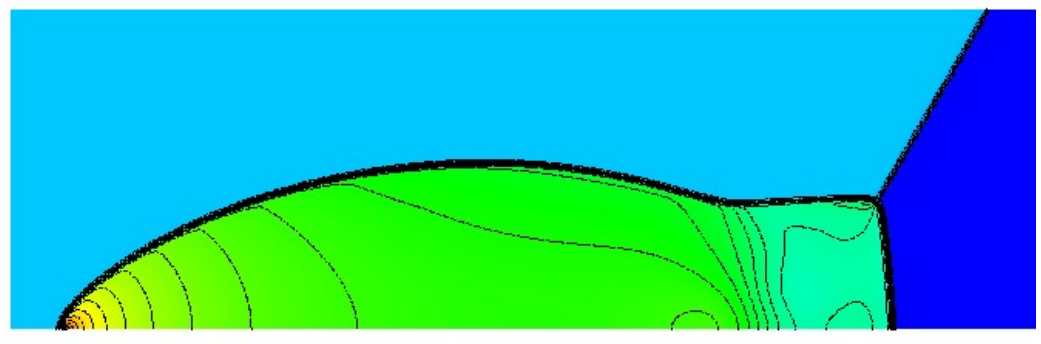

(c) RUS- $P_{1}$

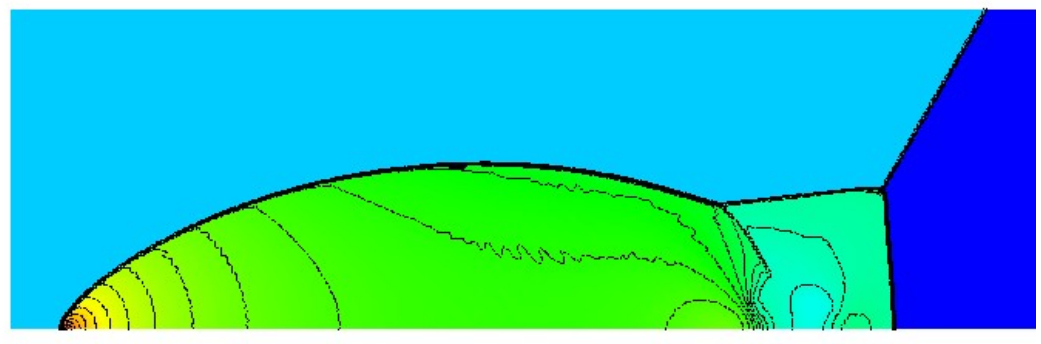

(d) HLL- $P_{1}$

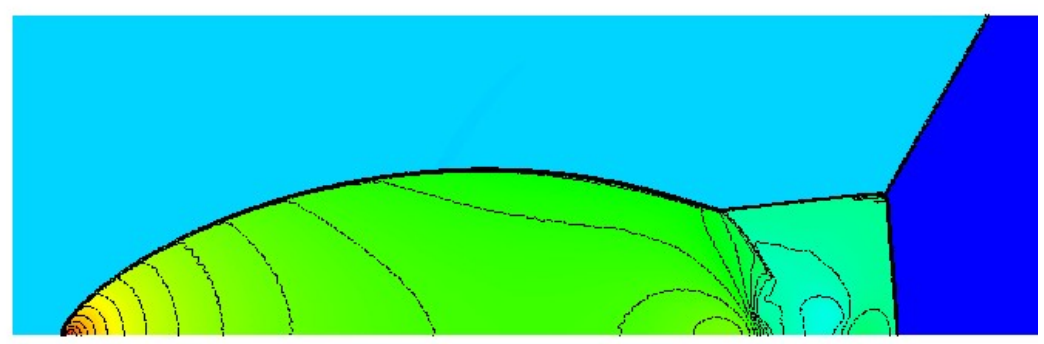

Figure 6: Double Mach reflection: $p$ at $t=0.2$, DG version, $h=\frac{1}{256}, \Delta t=10^{-4}$. 


\subsection{Noh problem}

In the second 2D example, we apply the constrained DG method to the Noh problem [24] which is regarded as a particularly difficult test for numerical methods. The computational domain for our $2 \mathrm{D}$ simulation is $\Omega=(0,1)^{2}$. In contrast to the previous examples, the adiabatic constant is $\gamma=5 / 3$. The exact solution is the infinite strength symmetric shock [17]

$$
\begin{aligned}
& \rho(x, y, t)= \begin{cases}1+t / \sqrt{x^{2}+y^{2}} & \text { if } \sqrt{x^{2}+y^{2}}>t / 3, \\
16 & \text { otherwise }\end{cases} \\
& \mathbf{v}(x, y, t)= \begin{cases}-(x, y) / \sqrt{x^{2}+y^{2}} & \text { if } \sqrt{x^{2}+y^{2}}>t / 3, \\
0 & \text { otherwise, }\end{cases} \\
& p(x, y, t)= \begin{cases}0 & \text { if } \sqrt{x^{2}+y^{2}}>t / 3, \\
16 / 3 & \text { otherwise. }\end{cases}
\end{aligned}
$$

We use this exact solution to define the initial condition and the external states for the HLL Riemann solver on the boundaries. The 2D simulation ends at $t=2.0$. According to Liska and Wendroff [17], many high-resolution schemes produce highly oscillatory results or simply crash in this test. Using the synchronized $(\rho, E, p)$ limiter and the new sequential $(\rho, E, k)$ limiter to constrain the DG-HLL- $P_{1}$ approximation, we obtained the results presented in Fig. 7. The snapshots of the density distribution at $t=2.0$ exhibit some ripples but the overall quality of the numerical solutions is acceptable.

\subsection{Smooth vortex}

In the last numerical example, we consider the isentropic vortex problem $[6,25]$ which admits a smooth analytical solution. The adiabatic constant for the ideal gas equation of state (4) is $\gamma=1.4$. Periodic boundary conditions are prescribed on the boundary of the domain $\Omega=(-5,5)^{2}$. The initial condition and exact solution at the final time $t=10.0$ are given by $[6,25]$

$$
\begin{gathered}
\rho_{0}=T_{0}^{\frac{1}{\gamma-1}}, \quad p_{0}=\rho_{0} T_{0}, \quad T_{0}=1-\frac{(\gamma-1) \beta^{2}}{8 \gamma \pi^{2}} e^{1-r^{2}} \\
\mathbf{v}_{0}=(1,1)+\frac{\beta}{2 \pi} e^{0.5\left(1-r^{2}\right)}(-y, x)
\end{gathered}
$$


(a)
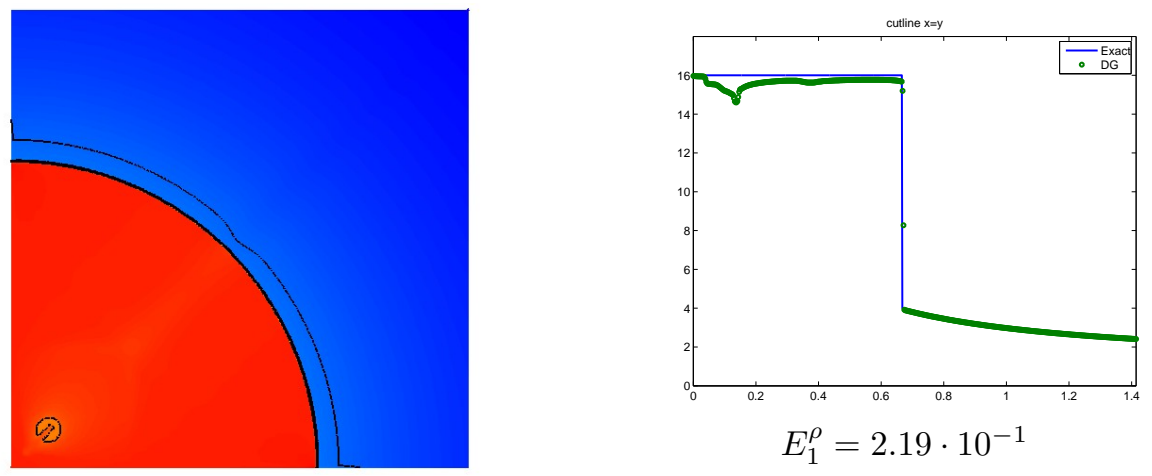

(b)
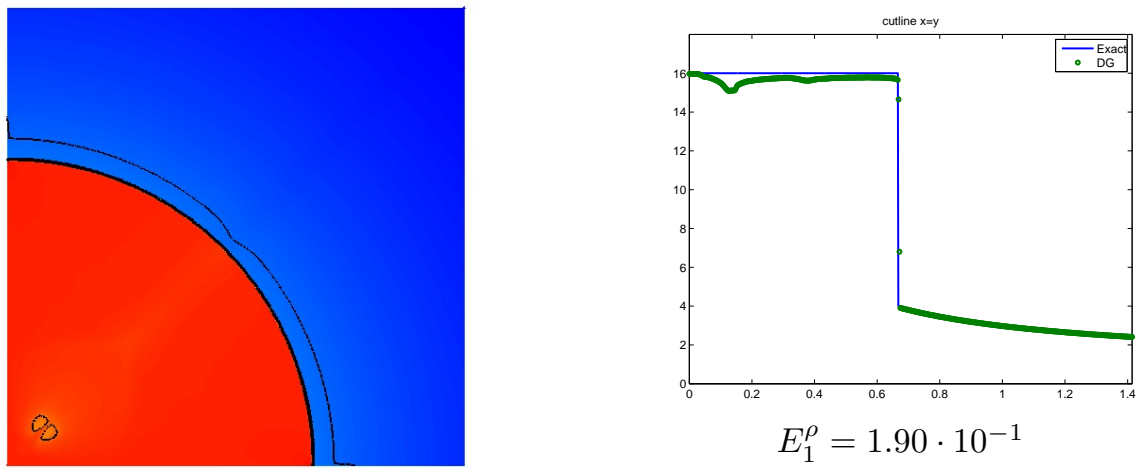

Figure 7: Noh problem: $\rho$ at $t=2.0, h=\frac{1}{512}, \Delta t=2.5 \cdot 10^{-4}$, DG-HLL version, (a) synchronized limiting of $(\rho, E, p)$, (b) sequential limiting of $(\rho, E, k)$.

where $\beta=5.0$ is the vortex strength and $r=\sqrt{x^{2}+y^{2}}$. We remark that $\beta$ should be $\beta^{2}$ in the initial condition for the temperature in reference [6].

The density snapshots displayed in Fig. 8 were calculated using the piecewiselinear DG-HLL approximation and the new sequential $(\rho, E, k)$ limiter. No visible changes in the symmetric shape of the density profile are observed as the center of the smooth vortex travels along the line $y=x$ with mean velocity $(1,1)$. The unconstrained DG solutions (not presented here) look the same. However, the grid convergence history presented in Tables 1 and 2 reveals that unnecessary limiting results in larger absolute errors and degrades the experimental order of convergence (EOC). This well-known drawback of local extremum diminishing limiters can be cured using smoothness indica- 
tors $[20]$ or hierarchical extensions $[10,11]$ of vertex-based slope limiters.

\begin{tabular}{|c|c|c|c|c|}
\hline$h$ & $P_{1}$, no limiter & $E O C$ & $P_{1}$, limited & $E O C$ \\
\hline $10 / 32$ & $0.215 \mathrm{e}+00$ & & $0.734 \mathrm{e}+00$ & \\
$10 / 64$ & $0.445 \mathrm{e}-01$ & 2.27 & $0.190 \mathrm{e}+00$ & 1.95 \\
$10 / 128$ & $0.894 \mathrm{e}-02$ & 2.32 & $0.767 \mathrm{e}-01$ & 1.31 \\
$10 / 256$ & $0.199 \mathrm{e}-02$ & 2.17 & $0.428 \mathrm{E}-01$ & 0.84 \\
\hline
\end{tabular}

Table 1: Smooth vortex: DG-HLL error $E_{1}^{\rho} \approx\left\|\rho-\rho_{h}\right\|_{L^{1}(\Omega)}$ and convergence rates.

\begin{tabular}{|c|c|c|c|c|}
\hline$h$ & $P_{1}$, no limiter & $E O C$ & $P_{1}$, limited & $E O C$ \\
\hline $10 / 32$ & $0.512 \mathrm{e}-01$ & & $0.208 \mathrm{e}+00$ & \\
$10 / 64$ & $0.104 \mathrm{e}-01$ & 2.30 & $0.536 \mathrm{e}-01$ & 1.96 \\
$10 / 128$ & $0.196 \mathrm{e}-02$ & 2.41 & $0.192 \mathrm{e}-01$ & 1.48 \\
$10 / 256$ & $0.412 \mathrm{e}-03$ & 2.25 & $0.961 \mathrm{e}-02$ & 1.00 \\
\hline
\end{tabular}

Table 2: Smooth vortex: DG-HLL error $E_{2}^{\rho} \approx\left\|\rho-\rho_{h}\right\|_{L^{2}(\Omega)}$ and convergence rates.

\section{Summary}

The limiting strategy introduced in this paper makes it possible to use individually chosen correction factors for different conserved variables and enforce local maximum principles for derived quantities in an efficient manner. The segregated treatment of the density field rules out any further smearing of contact discontinuities in the process of subsequent corrections. The use of compatible low-order approximations to density-weighted variables results in automatic adjustments of all conserved quantities after the density limiting step. This remarkable feature provides intrinsic synchronization of limited antidiffusive corrections without the need to use synchronized correction factors derived from a system of coupled inequality constraints under worst-case assumptions. The proposed methodology guarantees positivity preservation for the pressure and unifies element-based limiting techniques for continuous and discontinuous Galerkin methods. The results presented in this paper were obtained using linear finite elements. We envisage that extensions to high-order Bernstein elements can be derived in the same way as the generalized FCT algorithms developed in $[2,20]$ for scalar conservation laws. 

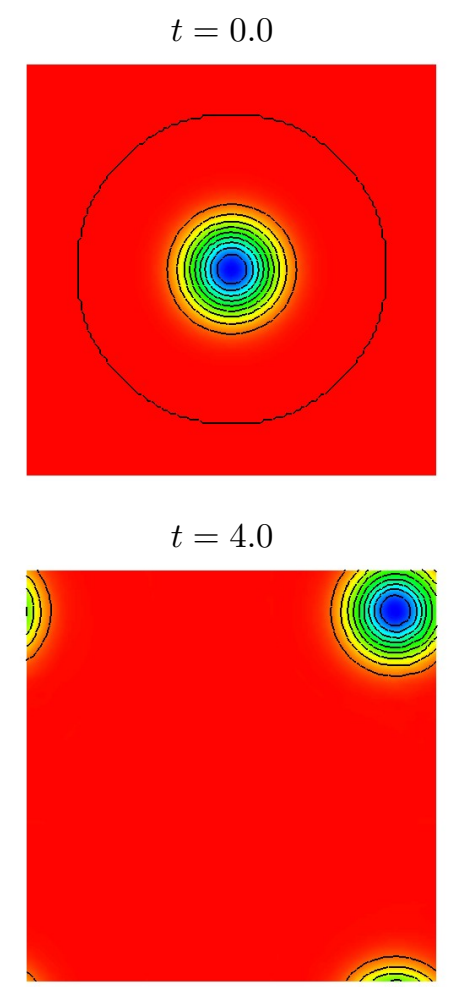

$$
t=8.0
$$

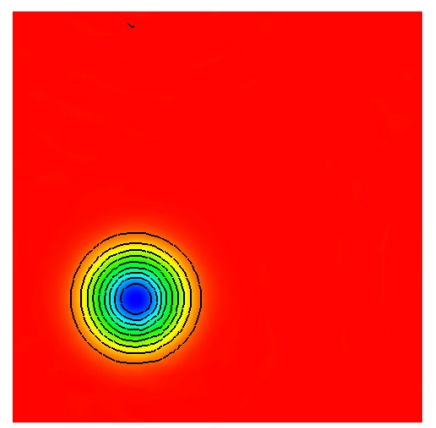

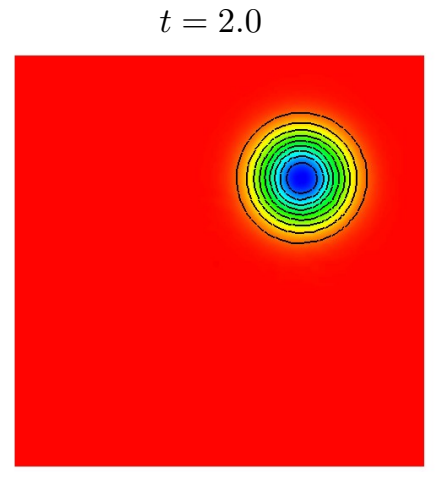

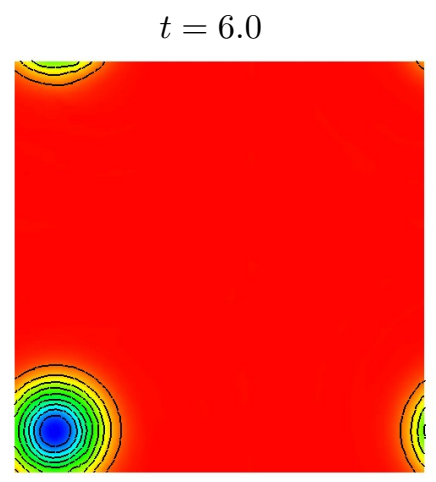

$$
t=10.0
$$

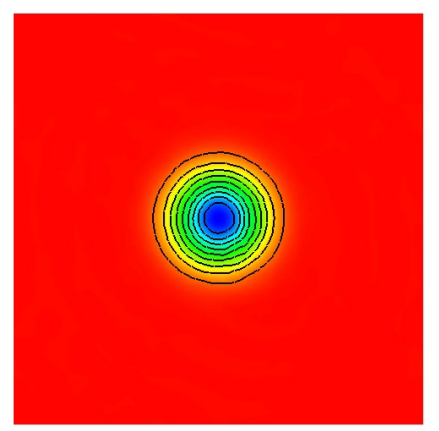

Figure 8: Smooth vortex problem: snapshots of the evolving density, $h=\frac{10}{128}, \Delta t=10^{-3}$, DG-HLL version, sequential limiting of $(\rho, E, k)$.

\section{Acknowledgments}

This research was supported by the German Research Association (DFG) under grant KU 1530/12-1 and under the auspices of the U.S. Department of Energy by Lawrence Livermore National Laboratory under Contract DE- 
AC52-07NA27344, LLNL-JRNL-732041. The authors would like to thank JProf. Sandra May (TU Dortmund University) for useful remarks and suggestions.

\section{References}

[1] R. Anderson, V. Dobrev, Tz. Kolev, R. Rieben, V. Tomov, High-order multi-material ALE hydrodynamics. LLNL preprint, 2017.

[2] R. Anderson, V. Dobrev, Tz. Kolev, D. Kuzmin, M. Quezada de Luna, R. Rieben, V. Tomov, High-order local maximum principle preserving (MPP) discontinuous Galerkin finite element method for the transport equation. J. Comput. Phys. 334 (2017) 102-124.

[3] T. Barth and D.C. Jespersen, The design and application of upwind schemes on unstructured meshes. AIAA Paper, 89-0366, 1989.

[4] P. Bochev, D. Ridzal, K. Peterson, Optimization-based remap and transport: A divide and conquer strategy for feature-preserving discretizations. J. Comput. Phys. 257 (2014) 1113-1139.

[5] C.J. Cotter and D. Kuzmin, Embedded discontinuous Galerkin transport schemes with localised limiters. J. Comput. Phys. 311 (2016) 363373.

[6] M. Dumbser, O. Zanotti, R. Loubère, S. Diot, A posteriori subcell limiting of the discontinuous Galerkin finite element method for hyperbolic conservation laws. J. Comput. Phys. 278 (2014) 47-75.

[7] S. Gottlieb, D. Ketcheson, C.-W. Shu, Strong Stability Preserving Runge-Kutta and Multistep Time Discretizations. World Scientific, 2011.

[8] S. Gottlieb, C.-W. Shu, and E. Tadmor, Strong stability-preserving highorder time discretization methods. SIAM Review 43 (2001) 89-112.

[9] D. Kuzmin, Algebraic flux correction I. Scalar conservation laws. In: D. Kuzmin, R. Löhner, S. Turek (eds), Flux-Corrected Transport: Principles, Algorithms, and Applications. Springer, 2nd edition, 2012, pp. 145-192. 
[10] D. Kuzmin, A vertex-based hierarchical slope limiter for $p$-adaptive discontinuous Galerkin methods. J. Comput. Appl. Math. 233 (2010) 30773085 .

[11] D. Kuzmin, Hierarchical slope limiting in explicit and implicit discontinuous Galerkin methods. J. Comput. Phys. 257 (2014) 1140-1162.

[12] D. Kuzmin and C. Lohmann, Synchronized slope limiting in discontinuous Galerkin methods for the equations of gas dynamics. Ergebnisber. Inst. Angew. Math. 541, TU Dortmund, 2016.

[13] D. Kuzmin, M. Möller, M. Gurris, Algebraic flux correction II. Compressible flow problems, In: D. Kuzmin, R. Löhner and S. Turek (Eds), Flux-Corrected Transport: Principles, Algorithms, and Applications, Springer, 2nd edition, 2012, pp. 193-238.

[14] D. Kuzmin, M. Möller, J.N. Shadid, M. Shashkov, Failsafe flux limiting and constrained data projections for equations of gas dynamics. $J$. Comput. Phys. 229 (2010) 8766-8779.

[15] R. Liska, M. Shashkov, P. Váchal, B. Wendroff, Optimization-based synchronized flux-corrected conservative interpolation (remapping) of mass and momentum for Arbitrary Lagrangian-Eulerian methods. $J$. Comput. Phys. 229 (2010) 1467-1497.

[16] R. Liska, M. Shashkov, P. Váchal, B. Wendroff, Synchronized flux corrected remapping for ALE methods. Computers \&3 Fluids 46 (2011) 312317.

[17] R. Liska and B. Wendroff, Comparison of several difference schemes on 1D and 2D test problems for the Euler equations. SIAM J. Sci. Comput. 25 (2003) 995-1017.

[18] J. Liu, J. Qiu, M. Goman, X. Li and M. Liu, Positivity-preserving Runge-Kutta discontinuous Galerkin method on adaptive Cartesian grid for strong moving shock. Numer. Math. Theor. Meth. Appl. 9 (2016) $87-110$.

[19] C. Lohmann and D. Kuzmin, Synchronized flux limiting for gas dynamics variables. J. Comput. Phys. 326 (2016) 973-990. 
[20] C. Lohmann, D. Kuzmin, J.N. Shadid and S. Mabuza, Flux-corrected transport algorithms for continuous Galerkin methods based on high order Bernstein finite elements. J. Comput. Phys. 344 (2017) 151-186.

[21] R. Löhner, K. Morgan, J. Peraire, M. Vahdati, Finite element fluxcorrected transport (FEM-FCT) for the Euler and Navier-Stokes equations. Int. J. Numer. Meth. Fluids, 7 (1987) 1093-1109.

[22] G. Luttwak and J. Falcovitz, Slope limiting for vectors: A novel vector limiting algorithm. Int. J. Numer. Methods Fluids 65 (2011) 1365-1375.

[23] S.A. Moe, J.A. Rossmanith, D.C. Seal, A simple and effective highorder shock-capturing limiter for discontinuous Galerkin methods. arXiv:1507.03024 [math.NA], 2015.

[24] W.F. Noh, Errors for calculations of strong shocks using an artificial viscosity and an artificial heat flux. J. Comput. Phys. 72 (1987) 78-120.

[25] C.-W. Shu, Essentially non-oscillatory and weighted essentially nonoscillatory schemes for hyperbolic conservation laws. NASA/CR-97206253, ICASE Report No. 97-65, 1997.

[26] G. Sod, A survey of several finite difference methods for systems of nonlinear hyperbolic conservation laws. J. Comput. Phys. 27 (1978) 1-31.

[27] P. Váchal and R. Liska, Sequential flux-corrected remapping for ALE methods. In: A. Bermudez de Castro, D. Gomez, P. Quintela, and P. Salgado (eds.) Numerical Mathematics and Advanced Applications (ENUMATH 2005). Springer, 2006, pp. 671-679.

[28] P.R. Woodward and P. Colella, The numerical simulation of two-dimensional fluid flow with strong shocks. J. Comput. Phys. 54 (1984) 115-173.

[29] S. T. Zalesak, The design of Flux-Corrected Transport (FCT) algorithms for structured grids. In: D. Kuzmin, R. Löhner, S. Turek (eds), Flux-Corrected Transport: Principles, Algorithms, and Applications. Springer, 2nd edition, 2012, pp. 23-66.

[30] X. Zhang and C-W. Shu, On positivity-preserving high order discontinuous Galerkin schemes for compressible Euler equations on rectangular meshes. J. Comput. Phys. 229 (2010) 8918-8934. 
[31] X. Zhang and C-W. Shu, Maximum-principle-satisfying and positivitypreserving high-order schemes for conservation laws: survey and new developments. Proc. R. Soc. A 467 (2011) 2752-2776. 